mitted, she complained of great pain in her back and abdomen, and the vomiting continued. The tumour was about the size of a small walnut, very firm and incompressible, and being evidently one of the class of hernige which is so much damaged by delay and the unnecessary use of the taxis and its auxiliaries, I deemed it proper to send for the surgeon of the week. Mr. Luke being absent from home, Mr. Critchett attended, and having examined the case, at once advised an operation, which was, after some hesitation, consented to. An incision was made, about an inch and a half in length, commencing an inch above the upper margin of the tumour; the subjacent cellular tissue and fascia were then divided down to the sac. Mr. Critchett passed his finger to the seat of stricture. (which was in this case Gimbernat's ligament, and returned the gut without opening the sac. Theré was a little arterial hæmorrhage; but as no vessel could be perceived, the wound was plugged with lint. The patient was then placed in bed, and ordered an opiate.

December 4th.- She has passed a farourable night. The lint was removed, and the edges of the wound brought together. She continued to go on well, with the exception of a little return of her rheumatism, and a slight attack of diarrhoa, until January 15th, 1851, when she was discharged cured.

The second case, which was under the immediate care of my colleague, Mr. Prater, presented some similar features. The patient was a woman, aged 47 , who had been the subject of hernia for four days only, having first perceived it while carrying some water up stairs, rollowing her occupation of a nurse. On examination, a tumour about the size of a bantam's-egg was found in the right groin, not very tender, but tense and unyielding. She complained of pain over the whole abdomen, and had vomited frequently during the day. The bowels had been relieved in the course of the morning. Mr. Curling, who saw her shortly after her admission, recommended the use of ice, and requested, that if after the lapse of a few hours, this produced no effect, Mr. Critchett should be called, as the latter gentleman had seen the patient previous to her coming to the hospital. The symptoms did not abate, and Mr. Critchett, on examining the patient, recommended an operation, which was at once proceeded with. After the preliminary steps, Gimbernat's ligament was divided, but the hernia could not be returned, and Mr. Critchett, on passing his finger backwards, found the obstruction to be a small band of condensed fascia, on freeing which, the gut was reduced without opening the sac. The after-treatment was much as usual, and the patient left the hospital cured, January 15th.

In each of these cases it wlll be perceived that the bowels were relieved a very short time before the operation was performed, and in neither was the sac opened. It is only, I think, by collecting a number of cases, that the relative value of operating with or without opening the sac, can be determined; and a wish to prove the superiority of the latter operation must, in some measure, be my apology for troubling you with this paper.

London Irospital, 1851.

P.S.-I find, by the record of operations, that Mr. Luike has operated on seventeen cases of femoral hernia since 1843. In fifteen of the cases the sac was not opened, and but two of them proved fatal.

\section{POISONING BY THE BITE OF AN ADDER.}

\section{Bx GEORGE LAMBERT, EsQ., London Hospital.}

Having observed in The Lancet a statement of a case of poisoning by the bite of an adder, I beg to forward the following outline of a similar case:-

George $\mathbf{R}-$, aged twenty-three, weaver, was admitted into the London Hospital, May 18, 1851, under the care of Mr. Adams. The patient states that while walking in Epping Forest, near Chigwell, he heard a rustling in the bushes, and on going to see what caused the noise, observed what he thought was a common snake. He attempted to catch it, and was bitten on the ring finger of the right hand. He was much alarmed at the time, and about twenty minutes afterwards vomited a large quantity of bitter yellow fluid; was much purged; had desire to micturate, but conld not; felt very faint and sleepy, and seemed as though he were deprived of sight. A countryman who was near the place applied some adder's fat and chewed ash-leaf to the finger, but the patient did not experience any benefit. Being unable to procure any conveyance to London, he was obliged to wait till a cart passed, which took him to Ilford, whence he came by rail to the hospital, which he reached about six hours after the injury had been inflicted.

A ppearance when admitted. - The patient feels cold and sick; great anxiety expressed in the countenance, and the pulse small. The finger was white, but little swollen; the back of the hand very much swollen; red lines reaching to the elbow; forearm not much swollen or discoloured, but rather tender. A wet bandage was applied to hand and forearm, and the extremity was placed upon an inclined plane. Ordered three grains of sesquicarbonate of ammonia and an ounce of camphor mixture every four hours, and four ounces of brandy to be given during the night.

19th.-Ten A.M.: Has not slept; suffers no pain; pulse improved; arm much swollen and ecchymosed, the discoloration extending to the axilla; the glands in that region also much swollen and inflamed. Apply soap-liniment to the axilla; continue the medicines and bandage; also envelop the extremity in flannel. Nine P.M.: Inflammation subsiding; ecchymosis extended down the side to the crest of the ilium; bowels not having been relieved, one scruple of a powder composed of calomel with jalap is ordered.

20 th.-Improving in health; ecchymosis deepening in colour. hue.

24 th \& 25th.-The same.

26 th.-Colour decreasing, and becoming browner.

28th.-The forearm and elbow feel very hard, and the patient complains of pain and stiffness on attempting to bend the elbow. Ordered the arm to be rubbed with soap-liniment. The discoloration and stiffiness gradually disappeared, and the patient was discharged cured, June 3.

The circumstances which are the most worthy of remark in this case are the extreme prostration of strength and the extensive ecchymosis, which exceeded in intensity of colour and extent anything I have ever seen.

\section{CASE OF}

COMPLICATED GUN-SHOT WOUND OF THE LEFT ARM AND SHOULDER,

FOLLOWED BY SECONDARY HAMORRHAGE FROM THE AXILLARY ARTERY, AND THE SUCCESSFUL ARPUICATION OF A LIGATURE TO THE LEFT SUBCLAVIAN ARTERY.

\section{Bx JOHN CRAWFORD, Esq.}

ASSISTANT-SURGEON HONOURABLE EAST INDIA COMPANY'S SERFICE, BOMBAY PRESIDENCY.

(Continued from p. 413.)

26th.-No re-appearance of the hæmorrhage; the patient passed a good night, and says his strength is considerably restored; appetite good; tongue white, but clean; no thirst or headache; pulse 118, firmer to the feel; countenance pale and somewhat anxious; wound over subclavian uniting kindly; removed the sutures this morning; old wound discharging bloody serum and unhealthy matter; left arm pale, but its temperature not diminished, and there is a slight purr or thrill in the radial artery, which occasionally amounts to slightly sensible pulsation; sensation in the hand and forearm remains as before the operation; no action of bowels. Ordered, infusion of senna, three ounces; gentian and quassia, of each one ounce and a half; tartrate of soda and potassa, half an ounce; tincture of senna, three drachms; solution of acetate of ammonia, six drachms. Mix: one ounce and a half to be taken every six hours until the bowels are opened. The mixture to be stopped so soon as one motion shall be produced.

$27 \mathrm{th}$.- The patient passed a good night, and the general symptoms are improved this morning. However, upon examination of the wound over the subclavian, I found that the discharge, though of a good colour, was rather thin, and the incision, which was united in its whole extent yesterday, had opened at two points, the edges of which were pale and flabby. The old wound had begun to resume a healthy action again; the arm still retained its warmth, and the same degree of sensation, and there was an occasional faint, but perceptible pulsation at the wrist, which was synchronous with the heart's action. There was also one good natural dejection, accompanied with tapeworm; no thirst; no headache; the skin soft and not much beyond the natural temperature; tongue moist, slightly furred; appetite good; pulse 120, quick, but soft. The fracture is quite disunited, and the extremity of the upper fragment projects through the wound; nor have I considered it safe or advisable, since the occurrence of the hamorrhages, to 
subject the arm to the restraint that would be required to keep the fracture in position. Should an attempt be yet made to preserve the arm, the projecting portion of bone at least appears doomed to excision.

Treatment.-The arm retained in an easy position, and lightly covered with cotton wool; wound carefully dressed, and the utmost caution enjoined regarding the wound over subclavian. Diet mild, but nutritious; to omit aperient mixture.

28th.--Passed a very wakeful night, though he had no pain in the arm; countenance pale and somewhat haggard; visible pulsation of carotids and subclavian; the edges of wound over subclavian separated in some points, pale, and secreting a thin discharge; the surface of old wound also looks flabby; some specks of blood observable on the gums, which are soft and spongy, and have for years been easily made to bleed. These appearances cause me much anxiety regarding the safe separation of the ligature. The pulse, however, is better, 108, and soft; tongue clean; appetite good; skin natural. Left arm still retains a good temperature, and there is a slight pulsation at the wrist as yesterday. Extracted five shot from the incision behind the shoulder. Same treatment, with diet a little more substantial.-Dix P.M.: Wound over subclavian discharged thin matter during the day, and there has been pain in it this afternoon; throbbing of carotids and subclavian very visible to the eye, as well as a strong bruit arterre perceptible to the most delicate touch; tingling, burning pain in the left hand. My anxiety to-night is much increased. Pulse 118. General symptoms as morning.

March 1st.-The night has passed over without any untoward event, and the patient has slept passably. Discharge from the seat of ligature improved, and the old wound looking florid and healthy. Throbbing of carotids not so visible, and the bruit arterre diminished in intensity. Pulse 104; more steady; temperature and pulse of left arm much as yesterday. Altogether, the general symptoms appear to be improved today, a change which I attribute to the more solid character of yesterday's diet. Same treatment continued.

2nd.-Passed a very good night; general symptoms same as, but slightly improved upon, yesterday. To repeat the tonic aperient mixture as on the 26 th ult., and same general treatment.

3rd.--Rested well during the night; general symptoms improving, with the exception of the tingling, burning pain in the hand. Bowels moved; pulse 108; left radial pulse somewhat more perceptible.

5th.-Passed a rather wakeful night; state of both wounds, however, still encouraging; pulse in left radial artery quite perceptible, but irregular, and not synchronous with heart's action.

7th.-Some increase of suppuration about shoulder, but old wound closing in at the edges, and presenting good granulations; pulse still frequent, 120 , with increased temperature of skin; no other febrile symptoms, however; bowels twice moved. Extracted seven shot from the opening in the posterior wall of the axilla. Ordered a slight reduction in diet.

8th. - Suppuration from shoulder still copious; extracted twenty-eight shot from the posterior opening in the axilla. Pulse still frequent, with bowels rather irritable. Ordered the following powder, to be taken at night:-Chalk and opium, eight grains; mercury and chalk, four grains; quinine, one grain and a half.

9th.-General symptoms as yesterday. Extracted thirtytwo shot from the opening in the axilla. To repeat the powder at night.

11th.-General symptoms the same. Copious discharge from superior incision, which appears to proceed from the shoulder-joint. Temperature and appearance of arm natural, and pulsation of radial artery more perceptible, but still irregular. Bowels twice moved; pulse 112. Repeat the powder at night.

13th.-Ligature came away safely this morning at seven o'clock, being in all sixteen days six hours from the period of its application. Pulse less frequent, 104, soft and regular; skin moist and cool; tongue clean; appetite good; bowels regular; temperature and circulation of left arm as before. No medicine prescribed.

14th.-Patient improving; extracted four shots.

19th.-Patient progressing favourably since separation of ligature, and the line of incision healing; discharge from old wound healthy, and very much diminished, its cavity also filling up with good granulations, and the edges contracting. There is a flow of synovia from the joint, but the circulation and temperature of the arm continue good. The extremity of the upper fragment still projects through the wound, covered on its outside only by integument. The lower fragment is attached to its inner surface by a firm granular mass, which appears to extend up to the inside of the capsule of the joint. It appears to me possible that by carefully removing the projecting portion, cicatrization and ossific union may probably yet be attained, and a more useful arm saved than by excising the whole of the upper fragment. The patient has excellent nights, and sat up out of bed to-day.

29 th.-The improvement has been steady and regular since last report. Wound over subclavian almost healed; old wound contracting, and discharge much diminished. A lamella of the upper fragment has exfoliated to-day. Discharge of synovia has ceased. General symptoms good.

A pril 4th.-A superficial lamella of necrosed bone brought away to-day from the inner aspect of the lower fragment, so that no more dead bone remains. The opposed surfaces of the two fragments are adhering by a pretty firm granular substance, but I am still doubtful about the attainment of ossific union. There is no further discharge of synovia.

15th.-The line of incision over the subclavian having been quite healed, and the patient duly prepared, I proceeded today to remove the projecting portion of the upper fragment. As its outer surface and a portion of its end were still covered with soft parts, it required a little dissection to completely expose the bone, so as to allow of its being properly sawn off.

This object I effected by first making an incision upwards and outwards from the superior corner of the wound, then another transversely outwards, pretty close to the end of the bone. The soft parts were then dissected carefully backwards, preserving even the granulations with them, so as to form a flap, of the shape of a truncated $\mathbf{V}$, with the base externally. This completely exposed the end of the bone, which was grasped with the fingers of the left hand, and the point drawn out pretty well, so as to allow of the application of a metacarpal saw. The section was then commenced from below and carried upwards, approaching towards the surface, until a complete wedge-shaped portion of bone was removed, of about $1 \frac{5}{5}$ of an inch in length, the base comprising the whole cylinder of the bone, and the apex running up to nothing. The outer edge of the bone was afterwards rounded with a bone forceps, and the flap replaced so as to cover the sawn surface. After all oozing of blood had ceased, the wound was dressed and the arm placed in a steady position. Upon this occasion, as upon that of the operation upon the subclavian, I felt much the want of the countenance and support of a professional brother; however, by the aid of a rather intelligent native hospital assistant, I succeeded in accomplishing the object $I$ desired. (See Fig. 2.)

FIG. 2.

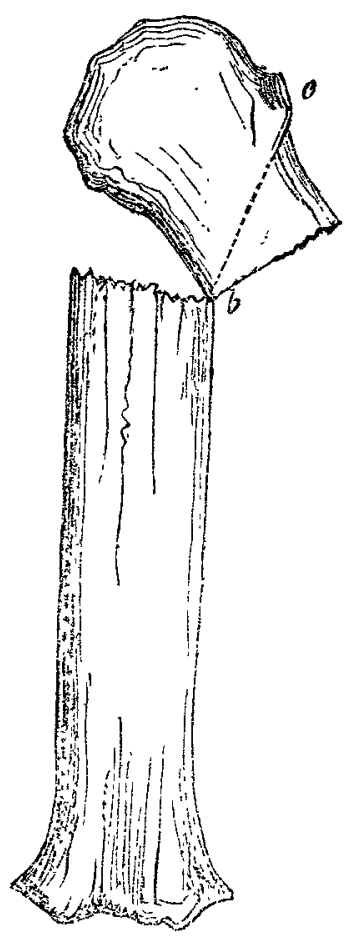

Fig. 2 shows the relation of the ends of the bone after it was considered inadvisable to persevere in retaining them in position, the superior fragment projecting through the wound, its tion, the superior frag cont prod line, $a, b$, indicates the line of excision of the wedge-shaped portion, upon the 15th of April.

25th.-Since the operation upon the 15 th the patient has been doing well, and the wound granulating and discharging 
healthy pas; strength of patient improving, and the general symptoms favourable. To-day I made an incision into a small collection of matter on the posterior surface of the shoulder, from which I extracted four shot.

May 8th.--The patient is now walking about, and the old wound healing steadily. I lave had a couple of gum splints constructed, which envelop the side of the chest, the shoulder, and arm down to below the elbow. By the aid of this shield, I am enabled to keep the fractured bone in a quiet, steady position, and am yet led to hope, that, by proper care, a pretty firm union of the bone may still be attained. The general health and strength of the patient are almost restored. Diet and general treatment, such as is calculated to promote the strength of the patient.

12th. - To-day the patient was embarked on board the steam frigate Auckland, for passage to Bombay. His strength is so far restored, that he was able, without fatigue, to walk some little distance to the beach. The arm retained in the gum splints.

22nd.-Last evening, the patient arrived safely at Bombay, from Bassadore. The wound all cicatrized to about the size of a shilling at its centre, and this healthy and closing in rapidly. The fracture is pretty firmly adherent, though it can scarcely be considered ossific as yet. There is much reason to hope, however. that the latter object will be yet attained. Health and strength quite re-established. The patient has been sent on shore to day to the Naval Sanatarium, under the charge of Assistant-Surgeon Reynolds, the port surgeon.

Aug. 8th.-A few days after the last report, I was hurriedly dispatched from Bombay to Aden, so that in the mean time I was debarred from ohserving the progress of my patient. Today, however, he arrived here upon his passage to England, and $I$ had an opportunity of examining him. He is in robust health. The gun-shot wound has quite healed over, and the union of the fracture has become perfectly firm and ossific. He has full power over the motions of the hand, forearm, and elbow-joint. The movements of the shoulder, as was to be expected, are of course yet feelle; but it is beyond all doubt, that ere the expiration of six months, he will have a very excellent arm. It is a matter of surprise to me, too, that notwithstanding the excision of the portions of humerus, and the present overlapping of the fragments, there is, upon the most careful measurement, only a shortening of the arm to the amount of an inch and a half.

Fra. 3.

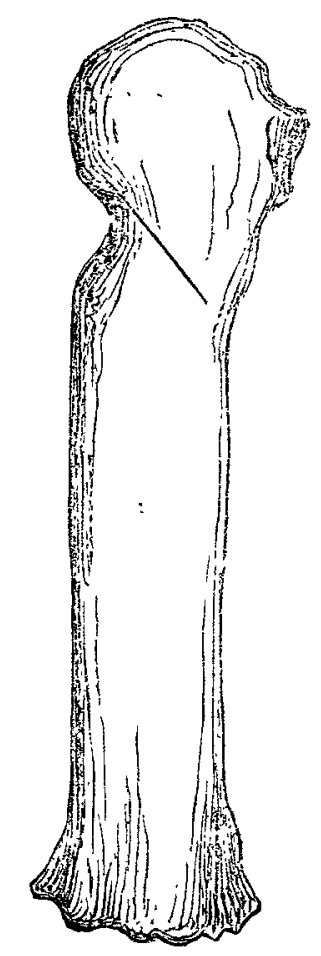

Fig. 3 is intended to convey my idea of how the bone has united, and what its present shape may be supposed to resemble.

(To be concluded in our next.)

New Medical College.-The Subscriptions now amount to nearly $£ 3000$.
CLINICAL REMARKS ON A CASE OF

ASTHMATIC BREATHING, OCCASIONED BY AN ANEURISM OF THE THORACIC AORTA.

By C. J. B. ALDIS, M.D.

$\mathrm{J}_{\mathrm{OHN}} \mathrm{J}-$, aged forty-five, residing at Pimlico, came under my care at the St. Paul and Barnabas Dispensary, on the 30th of August last.

I always visited him at home in consequence of the severe embarrassment in his breathing. He was rather tall and well-proportioned, the chest being fully developed. The first symptom which struck me on entering his room was a very loud tracheal râle during inspiration; his countenance, hands, and body were remarbably exsanguine, the hands almost white. He seemed to be afraid of the least exertion, lest the dyspnoea should be increased. The pulse feeble and regular; very little cough; no expectoration; in fact, there was no expectoration until towards the termination of the complaint. The chest was resonant on percussion, and no bruit could be detected over the cardiac region. The very loud sonorous inspiration, however, was sufficient to mask any slight murmur which might have existed. He stated that at the commencement of his illness he had lost his voice, which had since returned. There was no impulsive action of the heart. Previous to this attack, he had lived with Lady $\mathrm{D}-$ as groom of the chamber, and before that at Wimbledon, where he slept in a damp room, to which he attributed his illness.

On first seeing this patient, one might naturally ask, was the dyspnoea entirely spasmodic? But the attacks were not sufficiently transient. Again, was it the extension of laryngitis to the mucous membrane of the trachea, causing permanent thickening? But there had been no cough at the commencement, and no expectoration. Did it arise from pulmonary disease? but the lungs were resonaut, indeed more resonant than natural, which might have led any one to suppose that they were somewhat emphysematons. "The cause evidently existed close to the trachea, for on applying the stethoscope in the front part of the neck the loudest râle was heard just below the larynx. From these circumstances it was supposed that a tumour of some kind pressed upon the trachea, causing mechanical obstruction to the respiration. On one occasion when I visited him, Sept. 27 th, a loud sonorous râle accompanied each inspiration, followed by a loud cooing sound during expiration. There was no orthopnoea, and the illness was said to have commenced about three months ago. He had been an outpatieut at St. George's Hospital for about a fortnight before I saw him, but only went there three times, and was obliged to go in a cab.

The treatment consisted of counter-irritation with sedatives, stimulants, inhalation, and mild aperients. The difficulty of breathing becoming worse, he sank very gradually, and died on the 29 th of September.

A post-mortem examination of the body took place on the 30 th of $B$ eptember, when the following appearances were detected:-Body remarkably pale; there was a deposit of fat under the shin, and the muscles were well coloured, contrasting very much with the external paleness. On opening the thorax, the lungs collapsed but little; there was no emphy. sema, and they were healthy, but contained more than the usual quantity of air; no effusion into the chest; the heart healthy as to its dimensions and valves, but somewhat flabby; the vessels contained much fluid blood, with a long yellowish coagulum in the aorta, containing dark-coloured blood. On detaching the vessels from the heart, a tumour was observed in the arch of the aorta pressing on the trachea, which was taken out with it, including the larynx. On passing the finger into the aorta, it entered the swelling, which proved to be an aneurismal pouch. These appearances satisfactorily explained the previous symptoms. Had the patient survived much longer, the probability is, that the aneurism would have burst into the trachea, and suffocated him. Sometimes it enlarges very much, causing absorption of the vertebræ and ribs, and a tumour is formed external to the chest, which has been mistaken for an abscess, showing the necessity of making a very careful examination before plunging a lancet into some swellings. Occasionally the aorta is merely dilated without any well-formed pouch. In this morbid specimen you observe a distinct arterial sac, which pathologists term an aneurism. The coats are all entire, in which case it is called atria aneurism. Occasionally the two inner coats give way, leaving the outer coat, which is called a false aneurism. Should another pouch be formed out of the external coat, it is said to be "mixed." If the blood pass between the internal and middle coats through an aperture, obliterating a part of the channel, 